\title{
Determination and Validation of Eflornithine Hydrochloride by (Rp-Hplc) Reversed Phase High Performance Liquid Chromatography
}

\author{
Amit Kumar', Vijender Singh², Praveen Kumar, \\ ${ }^{1}$ Department of Pharmaceutical analysis, NKBR College of Pharmacy \& Research Centre, Meerut-245206, India \\ ${ }^{2}$ Department of Pharmaceutical analysis, BBS Institute of Pharmaceutical \& Allied Sciences, Greater Noida-201306, India \\ ${ }^{3}$ Department of Pharmaceutical Chemistry, S. D. College of Pharmacy and Vocational Studies, Muzaffarnagar- 251001, India \\ *Corresponding author: praveensha77@gmail.com
}

Received May 05, 2013; Revised December 02, 2013; Accepted December 11, 2013

\begin{abstract}
A simple highly sensitive, rapid, precise, accurate and specific RP-HPLC method was developed for quantification of eflornithine hydrochloride (2-difluoromethyl-DL-ornithine; DFMO) in its commercial formulation. In this RP-HPLC method, the separation was performed using BDS Hypersil $5 \mu$ C18 (150 X $4.6 \mathrm{~mm})$ column at room temperature by using methanol: $2 \%$ of Glacial acetic acid in water $(80: 20 \mathrm{v} / \mathrm{v})$ as mobile phase. The flow rate was $0.8 \mathrm{~mL} \mathrm{~min}^{-1}$ with UV detection at $290 \mathrm{~nm}$. The retention time of DFMO was 4.3. Linearity was observed over concentration range of $50-100 \mu \mathrm{gL}^{-1}$ for DFMO. The LOD and LOQ were found to be $0.008438 \mu \mathrm{g} \mathrm{mL}^{-1}$ and $0.028126 \mu \mathrm{g} \mathrm{mL}^{-1}$ respectively. The accuracy of the proposed method was determined by recovery studies and found to be $100.5 \%$ for DFMO. The proposed method was validated for ICH guidelines like linearity, limit of detection, accuracy, precision, ruggedness, robustness, and system suitability.
\end{abstract}

Keywords: eflornithine hydrochloride (DFMO), Validation, ICH guidelines, HPLC

Cite This Article: Amit Kumar, Vijender Singh, and Praveen Kumar, "Determination and Validation of Eflornithine Hydrochloride by (Rp-Hplc) Reversed Phase High Performance Liquid Chromatography.” American Journal of Pharmacological Sciences 1, no. 5 (2013): 90-95. doi: 10.12691/ajps-1-5-4.

\section{Introduction}

Difluoromethylornithine (DFMO; eflornithine hydrochloride) is a suicide inhibitor of the polyamine biosynthesis enzyme ornithine decarboxylase (ODC), one of the key enzymes in the polyamine biosynthetic pathway $[1,2]$. The drug was originally developed for use in cancer and is in phase III clinical trials for its use in preventing recurrence of superficial bladder cancer. It has been used as antiprotozoal agent in the treatment of meningoencephalic stage of trypanosomiasis caused by Trypanosoma brucei gambienze (African trypanosomiasis) $[3,4]$. DFMO currently is in development and testing for its anti inflammatory activity. DFMO $13.9 \%$ cream is used to inhibit growth and reduce the amount of facial hair in women [5].

A number of analytical methods have been reported for measuring DFMO in biological fluids and tissue extracts. These methods involve HPLC techniques [6,7,8]. The HPLC techniques currently available for the quantification of DFMO in biological fluids involve either pre or post column derivatization with UV or fluorescence detection $[9,10]$ and LC carried out by evaporative light scattering detection [11]. Few methods have been reported in the literature for the analysis of DFMO including spectrophotometry [12,13].

Regarding all the above mentioned, we decided to develop a newer RP-HPLC method suitable for determination of DFMO. The chemical structure of DFMO is presented in Figure 1.<smiles>NCCCC(N)(C(=O)O)C(F)F</smiles>

Figure 1. Chemical structure of eflornithine hydrochloride

Since there is no RP-HPLC analytical method for the determination of DFMO in the pharmaceutical formulations described in the literature. Therefore, the aim of this work was to develop and validate such a method that was newer, simpler, precise, sensitive, selective, economic, rapid and accurate.

In pharmaceutical industry the analysis of pharmaceuticals is an integral and increasingly important part of an overall drug development process. Therefore, rapid and simple methods for routine analysis and quality control of commercial formulations is desirable. Hence, in the present study, new, simple and selective reverse phase high performance liquid chromatography (RP-HPLC) method used for the determination of DFMO drug in commercially available pharmaceutical preparation was developed. 


\section{Materials and Methods}

\subsection{Apparatus and Software}

The HPLC (Shimadzu class LC-10A, including pump LC-10AT, SPD-10A UV-VIS detector, Japan) and Hamilton syringe (all from Shimadzu, Kyoto Japan) were used. The separation was achieved on a BDS Hypersil $5 \mu$ C18 (150 X 4.6mm) with UV detection at $290 \mathrm{~nm}$. Analytical weighing balance (Shimadzu AUX 200), sonicator (SONICA 2200MH), vacuum pump (model XI 5522050 of Millipore), millipore filtration kit for solvents and sample filtration were used throughout the experiment. The LC solution software-multiple channel was used for acquisition, evaluation and storage of chromatographic data.

\subsection{Reagents and Chemicals}

DFMO obtained as a gift sample (Wintac Pvt. Ltd. Bengaluru, India) and certified to contain 99.6\% purity. The drug is used without further purification. All the solvents used in analysis were of HPLC grade.

\subsection{Preparation of Solutions}

\subsubsection{Standard Solution of Eflornithine Hydrochloride}

Stock solution of DFMO was prepared at a concentration of ( $1 \mathrm{mg} \mathrm{mL}^{-1}$ ) by dissolving the accurate weighed amount in a definite volume of methanol to get the required concentration. Dilute solutions were prepared by accurate dilution from stock solution to get the desired concentrations.

\subsubsection{Preparation of Mobile Phase}

The mobile phase was prepared by mixing of glacial acetic acid (2\%) and methanol in the ratio 80:20 v/v.

\subsubsection{Pharmaceutical Sample Solution (from Formulation)}

Sample Ornidyl (1 mg mL ${ }^{-1}$ ) solution (label claimed, each fill volume of the vial contains $100 \mathrm{ml}$ and each $\mathrm{ml}$ contains $200 \mathrm{mg}$ ) was prepared by pipetting out 10 $\mathrm{ml}$ of the contents of the vial and geometrically diluted with methanol to get the desired concentration.

The solutions were sonicated for 10 mins and filtered into a $100 \mathrm{ml}$ volumetric flask through Whatman filter paper (no. 44). The residue was washed 3 times with 10 $\mathrm{ml}$ of methanol, and then the volume was made up to 100 $\mathrm{ml}$ with the same solvent. The proposed RP-HPLC method was applied and the concentration of each component in the formulation was determined. The results obtained were shown in Figure 2.

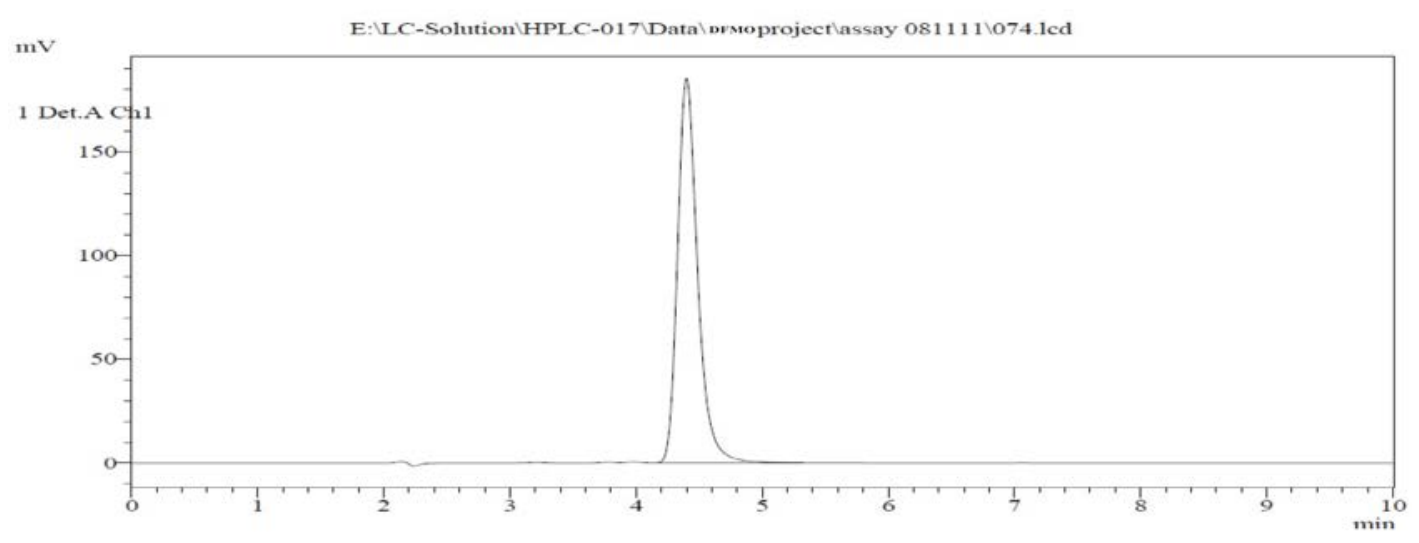

Figure 2. Chromatogram showing Retention Time $\left(\mathrm{R}_{\mathrm{t}}\right)$ of $50 \mu \mathrm{g} \mathrm{ml} \mathrm{l}^{-1}$ of DFMO (4.3 mins)

\subsection{Chromatography}

The mobile phase $2 \%$ of glacial acetic acid and methanol in the ratio $80: 20 \mathrm{v} / \mathrm{v}$ was selected, because it was found that it ideally resolved the peak with retention time (RT) 4.3 min for DFMO as shown in Figure 1. Wavelength was selected by scanning standard drug over a wide range of wavelength from $200 \mathrm{~nm}$ to $400 \mathrm{~nm}$. The component showed reasonably good response at $290 \mathrm{~nm}$.

\subsection{Validation of RP-HPLC Method}

Validation of an analytical method is the process to establish by laboratory studies that the performance characteristic of the method meets the requirements for the intended analytical application.

\subsubsection{Linearity}

The linearity of the proposed HPLC method for determination of DFMO was evaluated by analyzing a series of different concentrations of standard drug. In this study six concentrations were prepared, ranging between 50-100 $\mu \mathrm{g} \mathrm{mL} \mathrm{mL}^{-1}$ of DFMO. Each concentration was repeated three times and obtained information on the variation in the peak area response ratio of the internal standard to pure analytes is presented in Figure 4. Area is plotted graphically as a function of analyte concentration. The linearity of the calibration graphs was validated by the high value of correlation coefficient, slope and the intercept value Figure 3. A linear relationship was obtained for DFMO in the range of $50-100 \mu \mathrm{gL}^{-1}$.

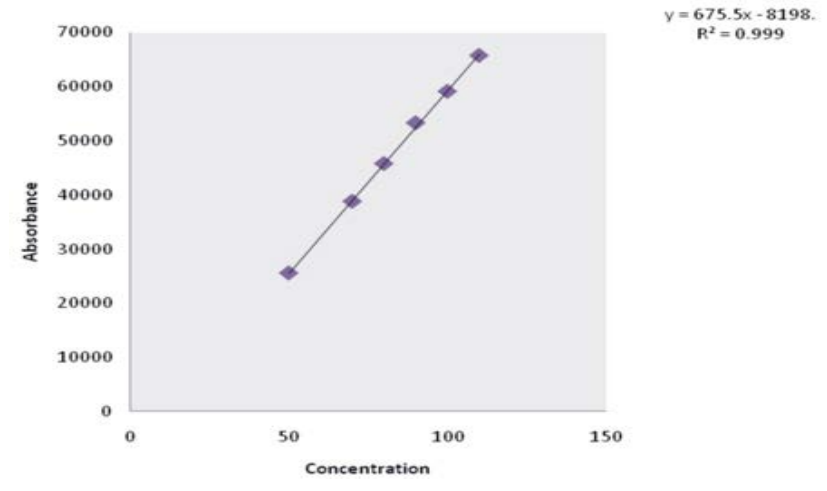

Figure 3. Linearity curves of DFMO at different concentration levels 


\subsubsection{Limit of Detection (LOD) and Limit of Quantification (LOQ)}

The limit of detection (LOD) and limit of quantitation (LOQ) were calculated according to ICH [14] recommendations where the approach based on the signalto-noise ratio. Chromatogram signals obtained with known low concentrations of analytes were compared with the signals of blank samples. A signal-to-noise ratio 3:1 and 10:1 was considered for calculating LOD and LOQ respectively. All analytical parameters obtained were shown in Table 1.

Table 1. Analytical Parameters of the Proposed RP-HPLC Method

\begin{tabular}{cc} 
Parameters & $\begin{array}{c}\text { RP-HPLC } \\
\text { (DFMO) }\end{array}$ \\
\hline Calibration range $(\mu \mathrm{g} \mathrm{mL}-1)$ & $50-100$ \\
Detection limit $(\mu \mathrm{g} \mathrm{mL}-1)$ & 0.008438 \\
Quantitation limit $(\mu \mathrm{g} \mathrm{mL}-1)$ & 0.028126 \\
Slope $(\mathrm{b})$ & 675.5 \\
Standard deviation of the slope (Sb) & 1.9 \\
Intercept (a) & 8198 \\
Correlation coefficient & 0.999 \\
Retention time & 4.3 \\
Theoretical plates \# & 6494.87 \\
Tailing factor & 1.3 \\
\hline
\end{tabular}
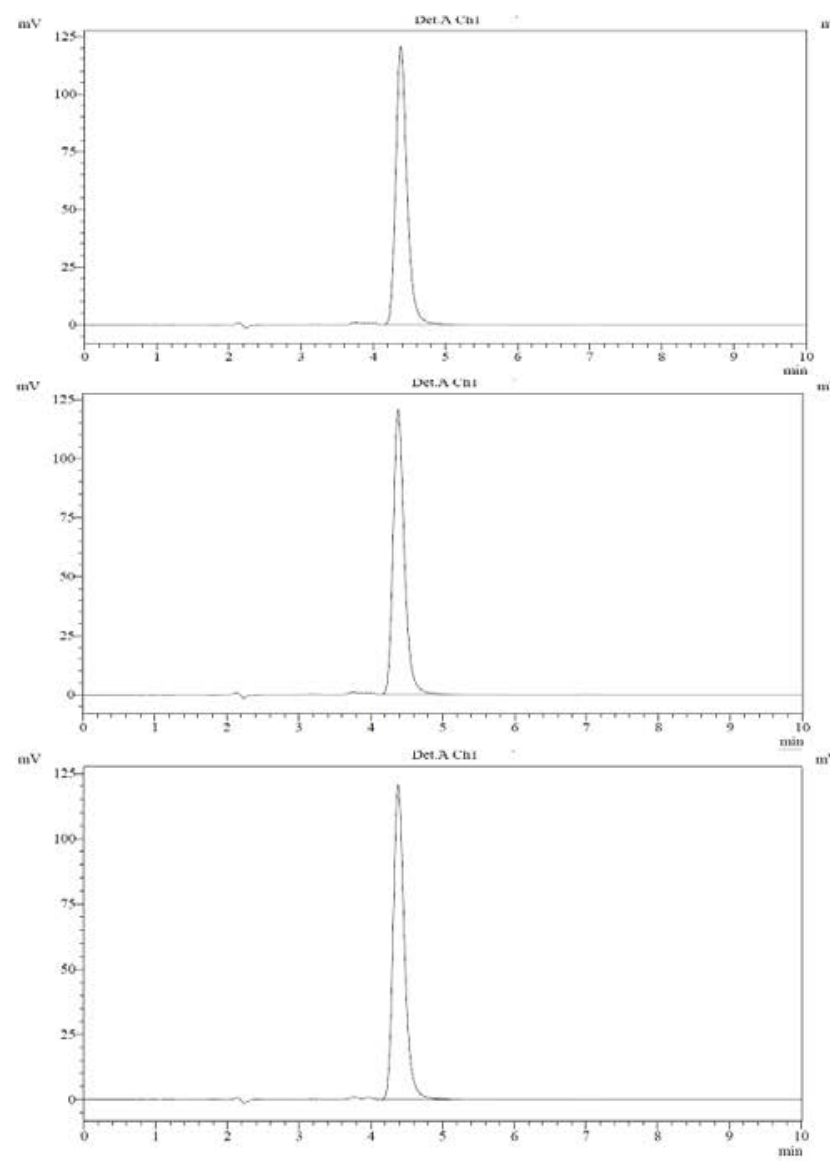

\subsubsection{Precision}

The precision of the analytical method was studied by analysis of multiple sampling of homogeneous sample. The precision expressed as \% RSD is given in Table 2 .

Table 2. Precision Study Results of Prepared Solutions HPLC

Validation parameter

\begin{tabular}{ccc} 
Repeatability $^{\mathrm{a}}$ & Peak area & Retention time \\
\hline $\begin{array}{c}\text { Eflornithine HCl } \\
\text { Intermediate precision }\end{array}$ & 1017852 & 4.35 \\
Eflornithine HCl $^{\mathrm{b}}$ & 1019308 & 4.33
\end{tabular}

${ }^{\mathrm{a}}$ Repeatability, three replicates of four concentration levels within-day bIntermediate precision, three replicates of four concentration levels between-days (3-days)

\subsubsection{Method Reproducibility}

The repeatability (within-day in triplicates) and intermediate precision (for 3 days) was carried out at four concentration levels for each compound. The obtained results within and between days trials are in acceptable range indicating good precision of the proposed method. The graphs were shown in Figure 4.
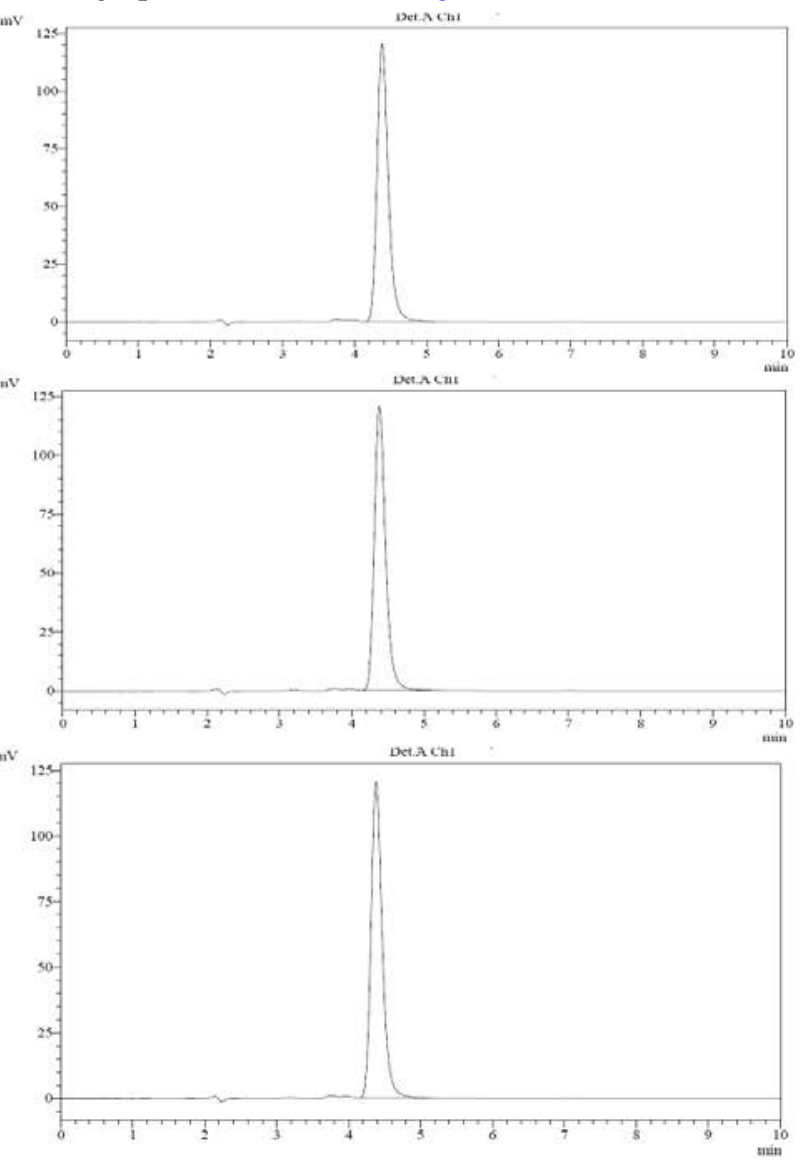

Figure 4. Linearity graphs of DFMO

\subsubsection{Accuracy}

The resulting mixtures were analyzed by the proposed HPLC method and the response obtained was plotted against the initial unknown concentration set at 0 as shown in Figure 5. The results obtained are compared with expected results. The excellent mean recoveries and standard deviation Table 3 suggested good accuracy of the proposed method and no interference from formulation exicipients.
Table 3. Application of Standard Addition Technique to the Analysis of Eflornithine

\begin{tabular}{cccc}
\hline \multirow{2}{*}{ Serial No } & \multicolumn{3}{c}{$\begin{array}{c}\text { Eflornithine HCl } \\
\end{array}$} \\
& Concentration in $\mu \mathrm{ml}^{-1}$ & \% recovery \\
& Added & HPLC \\
\hline 1 & 50 & 25 & 99.1 \\
2 & 50 & 50 & 100.5 \\
3 & 50 & 75 & 102.2 \\
\hline *Average of three experiments
\end{tabular}




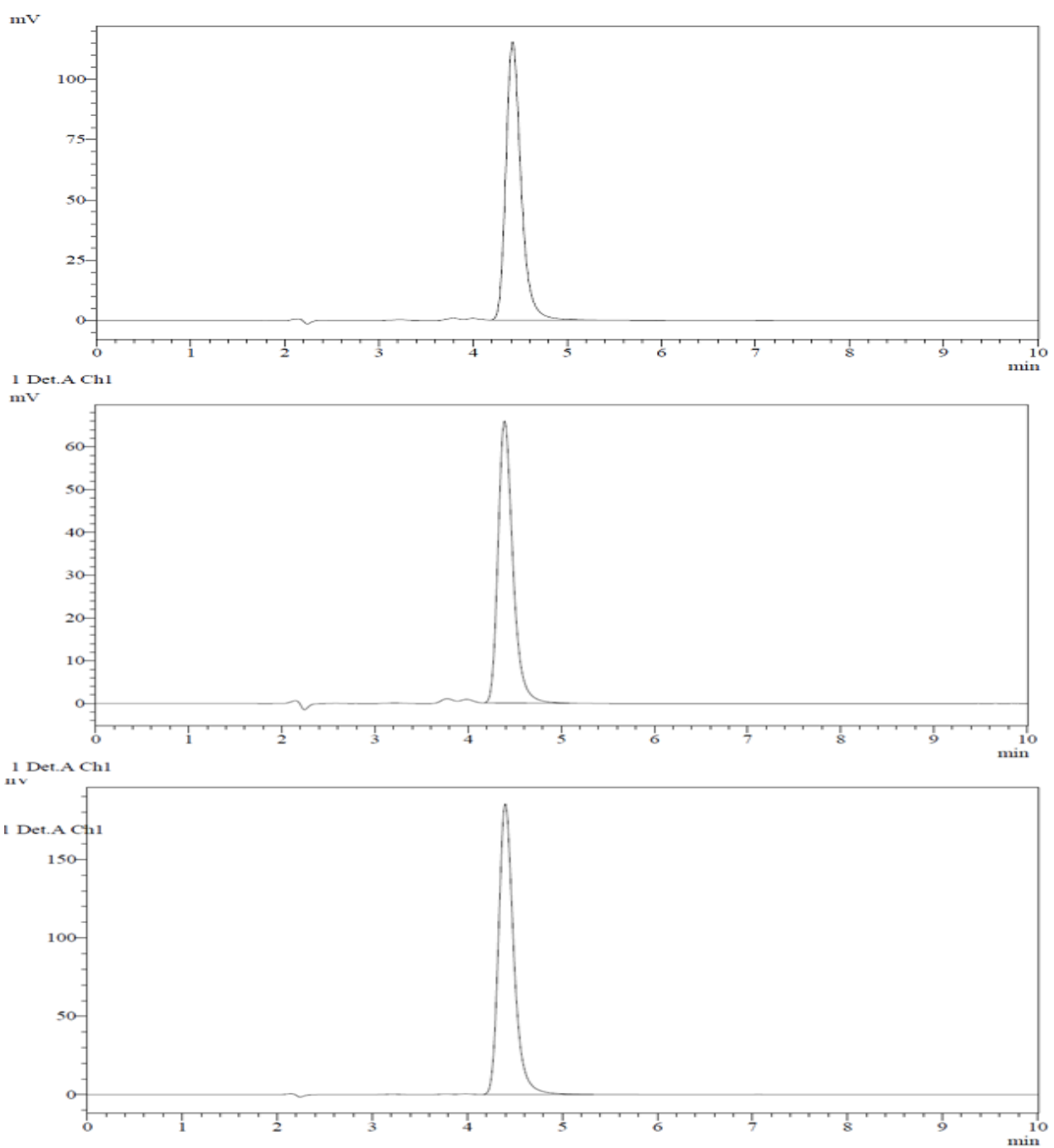

Figure 5. HPLC Chromatograms of DFMO in Eflornithine vials for Accuracy studies

\subsubsection{Ruggedness}

The assay of DFMO was performed by using different condition, different analyst, and different dates. As the results were within the acceptance limit, the proposed method is found to be rugged.

\subsubsection{Robustness}

The robustness of the proposed HPLC method was assessed for peak resolution and symmetric factor Table 4. The parameters investigated at column temperature $( \pm$

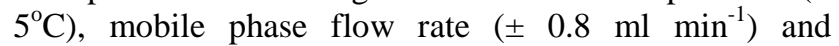
detection wave length $( \pm 5 \mathrm{~nm})$.

The robustness of the method shows that there were no marked changes in the chromatographic parameters, which demonstrates that the method developed was robust.

Table 4. Robustness of Chromatographic Method

\begin{tabular}{|c|c|c|}
\hline \multirow{2}{*}{ PARAMETERS } & \multicolumn{2}{|c|}{ (DFMO) } \\
\hline & Rt & Area \\
\hline \multirow{3}{*}{$\begin{array}{c}\text { Original } \\
\text { Column temperature }\left(35^{\circ} \mathrm{C} \pm 5^{\circ} \mathrm{C}\right)\end{array}$} & 4.358 & 1308639 \\
\hline & 4.367 & 1314236 \\
\hline & 4.321 & 1336321 \\
\hline \multirow{2}{*}{$\begin{array}{l}\text { Mobile phase flow rate } \\
\left(0.8 \pm 0.1 \mathrm{ml} \mathrm{min}^{-1}\right)\end{array}$} & 3.611 & 1328428 \\
\hline & 4.809 & 1353768 \\
\hline \multirow{2}{*}{$\begin{array}{l}\text { Detection wave length } \\
\quad(290 \pm 5 \mathrm{~nm})\end{array}$} & 4.324 & 1345212 \\
\hline & 4.332 & 1326612 \\
\hline
\end{tabular}

\subsubsection{System Suitability}

A solution of $50 \mu \mathrm{g} \mathrm{mL}^{-1}$ of DFMO (in triplicate) was prepared and same was injected, then the system suitability parameters were calculated from the following chromatogram Figure 6. The results obtained are represented in Table 5.

Table 5. Results of System Suitability Parameters

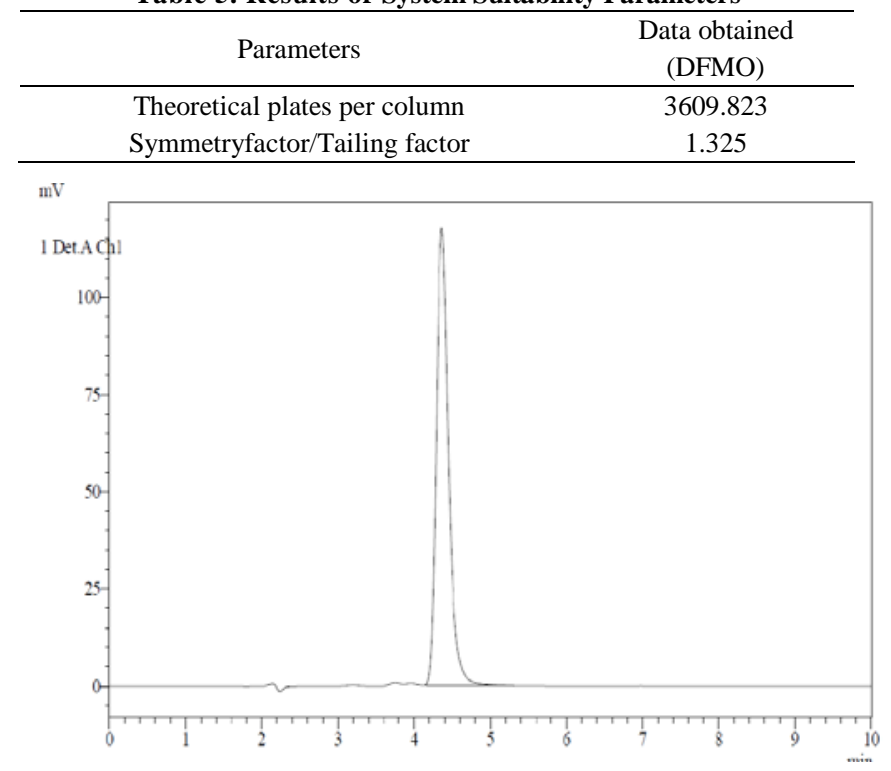

Figure 6. Chromatogram showing system suitability 


\subsubsection{Results of Analysis of Commercial Formulation}

The values of \% recovery from formulation as shown in the Table 6 are found to be very close to the label value of commercial pharmaceutical formulation. It shows that the method is applicable for determination of DFMO from the formulation.

Table 6. Results Obtained for the Pharmaceutical Samples by Using Formulation

\begin{tabular}{ccc}
\hline Vial & $\begin{array}{c}\text { Concentration in } \\
\mu \mathrm{gL}^{-1}\end{array}$ & \% recovery \\
\hline Ornidyl & 200 & 100.5 \\
\hline Ornidyl label claim: (200 mg of Eflornithine $\mathrm{HCl}$ per vial)
\end{tabular}

\section{Results and Discussion}

RP-HPLC method developed was found rapid, simple, precise, accurate and economical for routine estimation of DFMO in commercial dosage forms. There is no official method for the estimation of DFMO.

In RP-HPLC method, the conditions were optimized to obtain good peak of eluted compound. Initially, various mobile phase compositions were tried. Mobile phase and flow rate selection was based on peak parameters (height, tailing, theoretical plates, capacity or symmetry factor, run time, resolution). The system with a ratio of $(80: 20 \mathrm{v} / \mathrm{v}$ methanol: $2 \%$ of glacial acetic acid in water) with 0.8 $\mathrm{mL} / \mathrm{min}^{-1}$ flow rate was quite robust.

The optimum wavelength for detection was $290 \mathrm{~nm}$ at which better detector response for the drug was obtained. The average retention time for DFMO was found to be 4.3 mins. System suitability tests were an integral part of chromatographic method. They were used to verify the reproducibility of the chromatographic system. To ascertain its effectiveness, system suitability tests were carried out on freshly prepared stock solutions. The calibration was linear in concentration range of 50-100 $\mu \mathrm{g}$ $\mathrm{mL}^{-1}$, with regression 0.999 intercept 8198 and slope 675.5 for DFMO. The low values of \% RSD indicated that method was precise and accurate. Mean recoveries were found in the range of 98 - $102 \%$. Sample to sample precision and accuracy were evaluated using five samples of five different concentrations, which were prepared and analyzed on same day. Day to day variability was assessed using five concentrations analyzed on three different days. These results showed the accuracy and reproducibility of the assay.

Ruggedness of the proposed method was determined by analysis of aliquots from homogeneous slot in different laboratories, by different analysts, using similar operational and environmental conditions. The \% RSD reported was found to be less than $2 \%$. The proposed method was validated in accordance with ICH parameters and applied for analysis of the same in marketed formulations.

\section{Conclusion}

Proposed study describes a new RP-HPLC method for the determination of DFMO. For routine analytical purpose it was desirable to establish method capable of analyzing large number of samples in a short time period with good accuracy and precision without any prior separation step. The method was validated and found to be simple, sensitive, accurate and precise. Percentage of recovery shows that the method was free from interference of the excipients used in the formulation. Therefore, the proposed method can be used for routine analysis of DFMO in the dosage form.

\section{Acknowledgements}

First author is highly thankful to IFTM University, Moradabad, India for Ph.D. registration and facilities. We thank Mr. S. P. Venkatesh Prasad, PEC College of Pharmacy, Bangalore, India, for his technical support and Wintac Ltd, Bangalore, India, for providing a gift sample of DFMO.

\section{Competing Interests}

None

\section{Abbreviations}

UV, ultra-violet; RP-HPLC, reverse phase high performance liquid chromatography.

\section{References}

[1] Merali, S. and Clarkson, A. B. Jr, "Polyamine content of Pneumocystis carinii and response to the ornithine decarboxylase inhibitor DL-alpha-difluoromethylornithine," Antimicrob. Agents Chemother. 40(4). 973-978. 1996.

[2] Clarkson, A. B. Jr., C J Bacchi, C. J., Mellow, G. H., Nathan, H. C., McCann, P. P. and Sjoerdsma, A, "Efficacy of combinations of difluoromethylornithine and bleomycin in a mouse model of central nervous system African trypanosomiasis,” Proc. Natl. Acad. Sci., U.S.A. 80(18). 5729-5733. 1983.

[3] Milord, F., Loko, L., Mpia, B. and Pepin, J. "Eflornithine concentrations in serum and cerebrospinal fluid of 63 patients treated for Trypanosoma brucei gambiense sleeping sickness," Trans. R. Soc. Trop. Med. Hyg, 87(4). 473-7, 1993.

[4] Pepin, J., Guern, C., Milord, F. and Schechter, P. J, "Difluoromethylornithine for arseno-resistant trypanosoma brucei gambiense sleeping sickness,” The Lancet., 330(8573). 1431-1433. 1987.

[5] Balfour, J. A. and McClellan, K, “Topical eflornithine,” Am. J. Clin. Dermatol, 2(3). 197-201. 2001.

[6] Cohen, J. L., Ko, R. J., Lo, A. T., Shields, M. D. and Gilman, T. M, "High-pressure liquid chromatographic analysis of eflornithine in serum,” J. Pharm. Sci., 78(2). 114-6. Feb. 1989.

[7] Huebert, N. D., Schwartz, J. J. and Haegele, K. D, “ Analysis of 2difluoromethyl-DL-ornithine in human plasma, cerebrospinal fluid and urine by cation-exchange high-performance liquid chromatography,” J. Chromatogr. A., 762(1-2). 293-8. Feb. 1997.

[8] Saravanan, C., Kumudhavalli, M. V., Kumar, M. and Jayakar, B, "A new validated RP-HPLC method for estimation of eflornithine hydrochloride in dosage form,” J. Phar. Res., 2. 1730-1731. 2009.

[9] Kilkenny, M. L., Slavik, M., Christopher, M. R. and Stobaugh, J. $\mathrm{F}$, "Plasma analysis of alpha-difluoromethylornithine using precolumn derivatization with naphthalene-2, 3-dicarboxaldehyde/CN and multidimensional chromatography," J. Pharm. Biomed. Anal., 17. 1205-1213. 1998.

[10] Jansson-Löfmark, R., Römsing, S., Albers, E. and Ashton, M, "Determination of eflornithine enantiomers in plasma by precolumn derivatization with $O$-phthalaldehyde- $N$-acetyl-Lcysteine and liquid chromatography with UV detection,” Biomed. Chromatogr. 24(7). 768-773. July 2010. 
[11] Malm, M. and Bergqvist, Y, "Determination of eflornithine enantiomers in plasma, by solid-phase extraction and liquid chromatography with evaporative light-scattering detection," $J$. Chromatogr. B. Analyt. Technol. Biomed. Life Sci., 846. 98-104. 2007.

[12] Kumar, A., Venkatesh, Prasad, S. P., Mohan, S. and Kumar, P, "Spectrophotometric determination of eflornithine hydrochloride as active pharmaceutical ingredient using 1, 2-naphthoquinone-4- sulfonate as the derivative chromogenic reagent,” Trade Sci. Inc., 7, 2008.

[13] Kumar, A., Venkatesh, Prasad, S. P., Mohan, S. and Singh, A. K, "Estimation of eflornithine hydrochloride by UV spectroscopy," Trade Sci. Inc., 8. 2009.

[14] Validation of Analytical Procedures, Methodology ICH Harmonised Tripartite Guideline, Having Reached Step 4 of the ICH Process at the ICH Steering Committee meeting on November 6, 1996. 